\title{
Quantifying dynamics and interactions of individual spurious low-energy fluctuators in superconducting circuits
}

\author{
S. E. de Graaf $\odot,{ }^{1, *}$ S. Mahashabde, ${ }^{2}$ S. E. Kubatkin, ${ }^{2}$ A. Ya. Tzalenchuk $\odot,{ }^{1,3}$ and A. V. Danilov ${ }^{2}$ \\ ${ }^{1}$ National Physical Laboratory, Hampton Road, Teddington TW11 OLW, United Kingdom \\ ${ }^{2}$ Department of Microtechnology and Nanoscience, MC2, Chalmers University of Technology, SE-41296 Goteborg, Sweden \\ ${ }^{3}$ Royal Holloway, University of London, Egham TW20 OEX, United Kingdom
}

(Received 21 December 2020; revised 25 March 2021; accepted 9 April 2021; published 7 May 2021)

\begin{abstract}
Understanding the nature and dynamics of material defects in superconducting circuits is of paramount importance for improving qubit coherence and parameter stability and much needed for implementing large-scale quantum computing. Here we present measurements on individual highly coherent environmental two-level systems (TLS). We trace the spectral diffusion of specific TLS and demonstrate that it originates from the TLS coupling to a small number of low energy incoherent fluctuators. From the analysis of these fluctuations, we access the relevant parameters of low energy fluctuators: dipole moments, switching energies, and, more importantly, interaction energies. Our approach opens up the possibility of deducing the macroscopic observables in amorphous glassy media from direct measurements of local fluctuator dynamics at the microscopic level—a route towards substantiating commonly accepted, but so far phenomenological, models for the decohering environment.
\end{abstract}

DOI: 10.1103/PhysRevB.103.174103

\section{INTRODUCTION}

All superconducting qubit designs suggested so far suffer from noise and parameter drift and associated decoherence. These detrimental effects are known to stem from the qubit coupling to two-level systems (TLS) associated with spurious material defects and present a major issue towards engineering large scale superconducting quantum circuits [1-5]. Great progress has been made in the last decade towards understanding the decoherence mechanisms by using qubits as natural probes of individual TLS [5-13], yet their physical origin and chemical nature remain unknown.

Complementary, superconducting resonators allow for electron spin resonance (ESR) spectroscopy of small volumes and very few spins [14] and in situ chemical identification of dilute paramagnetic material defects [15]. This provides a route for straightforward evaluation of the effects of fabrication and surface treatments on defect density and composition [15-18]. However, resonators as probes of TLS were so far mainly used to reveal ensemble effects over a broad parameter range [5,15,16,19-22]. Detection of individual TLS in a superconducting resonator was first demonstrated with an embedded parallel plate capacitor [23,24], although this method does not differentiate between surface and

\footnotetext{
*sdg@npl.co.uk
}

Published by the American Physical Society under the terms of the Creative Commons Attribution 4.0 International license. Further distribution of this work must maintain attribution to the author(s) and the published article's title, journal citation, and DOI. bulk TLS, the former being most ubiquitous in qubits [10].

The detection of individual surface TLS with planar resonators was only recently demonstrated $[21,25,26]$. Together with increased loss, the TLS-resonator coupling causes a dispersive shift of the resonator frequency; the most prominent shift comes from near-resonant TLS with relaxation times on par or superior to that of the resonator itself. Such TLS should be treated as quantum coherent systems. The TLS-induced dispersive shift per se does not induce noise in resonators or qubits; it is the temporal instability of TLS parameters which is detrimental. Having energy level splittings $U_{\mathrm{TLS}}>k_{\mathrm{B}} T$, the near-resonant TLS are not subjected to thermal fluctuations and their parameter instability is attributed to interaction with much more abundant low energy fluctuators with $U_{\mathrm{TLF}}<$ $k_{\mathrm{B}} T$. These low-energy fluctuators (commonly referred to as TLF) have fast relaxation rates, behave as classical two-state systems, and constitute the fluctuating environment for the higher energy coherent TLS [5,27]. The fluctuations in the TLF bath are responsible for the instability of TLS and therefore comprise the ultimate source of noise and decoherence in quantum devices $[3-5,16,19]$. The TLS-TLF hierarchy is illustrated in Fig. 1(b).

As for their physical nature, the resonant TLS are usually assumed to be atomic scale tunneling systems, i.e., atoms in a double well potential, likely hosted by some lattice imperfection or surface adsorbates [5,10], but other origins have also been suggested $[21,28,29]$. The microscopic nature of classical TLFs is even more elusive. Only in one previous setting has an individual TLF (coupled to a TLS) been studied in a qubit setup [6,30]. The most celebrated model for the TLF medium is the standard tunneling model (STM) [31] which aims to derive the observed ensemble averaged properties 
(a)

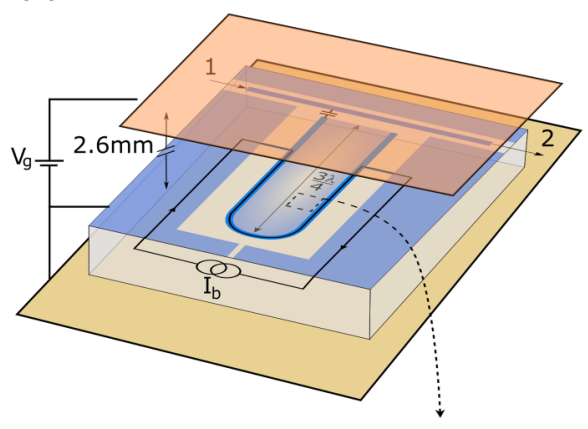

(b)

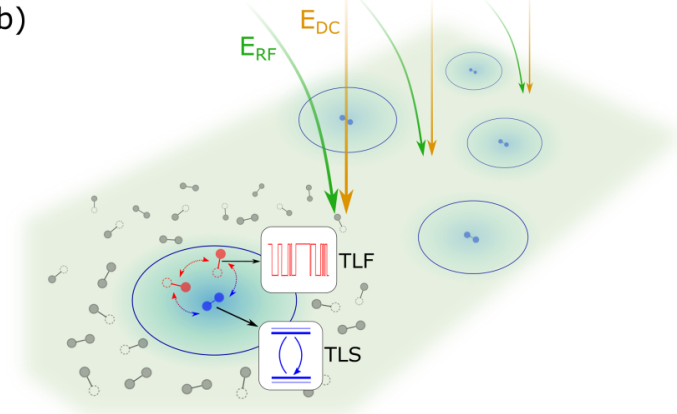

FIG. 1. Planar superconducting resonator as a sensor of fluctuating environmental defects. (a) Sketch of the sample setup: Tuning resonator frequency (via the applied current $I_{\mathrm{b}}$ ) and external electrostatic gate $V_{\mathrm{g}}$ allow us to select individual coherent TLS for study. The resonator is read out by measuring the transmission $S_{21}$ between ports 1 and 2. (b) The TLS (blue) couples to a few neighboring incoherent TLFs (red) within the TLS interaction volume (blue circles). The random switching of TLFs causes the telegraphlike fluctuation of TLS excitation energy. The larger bath of background TLFs (gray) affects the TLS linewidth. The TLS couple to the resonator via the microwave electric field $E_{\mathrm{RF}}$, and the DC electric field $E_{\mathrm{DC}}$ arising from the applied gate voltage $V_{\mathrm{g}}$ allows us to select specific TLS to be in resonance with the resonator.

of decohering media from a phenomenological description of individual two-level fluctuators and their parameters. As such, the STM (and modifications thereof [27,32,33]) does not provide insight into the physical origin of TLS/TLF, nor into the microscopic properties of individual TLS, instrumental for procedures to improve and understand material properties of low temperature condensed matter systems [34]. A microscopic theory of amorphous glassy media requires understanding of the origin and dynamics of individual TLS and their TLF environment, and in particular of their interactions.

Here we experimentally develop this approach in the specific case of superconducting devices and associated materials. We employ kinetic-inductance frequency tunable planar resonators $[25,35]$ in combination with external electrostatic gating as a versatile platform to resolve individual TLS and to track their dynamics. Such a coupled resonator-TLS system becomes a sensitive probe for the study of individual TLF in a wide temperature range from 10 to $>300 \mathrm{mK}$ and provides access to TLF-TLF dynamics and interaction energies. We reveal direct evidence of two interacting TLFs, sensed through the same TLS.

\section{EXPERIMENT}

The results were obtained with a planar superconducting resonator (made from a $\mathrm{NbN}$ thin film on a sapphire substrate). The resonator is made frequency tunable via its kinetic inductance by application of a small direct current bias $(<1 \mathrm{~mA})$. Details about resonator design and fabrication can be found in Ref. [25]. The resonators are cooled down to a base temperature of $10 \mathrm{mK}$ in a well filtered dilution refrigerator. The bias current is fed to the resonator through low-pass filtered lines and back to the ground (via a $50 \Omega$ termination) at the $4 \mathrm{~K}$ stage of the fridge. A detailed description of the experimental setup can be found in Ref. [21]. An additional and essential aspect of the present setup is an electrostatic gate located in the sample enclosure, separated from the sample by approximately $2.6 \mathrm{~mm}$, to control the TLS energy [10,12]. The sample setup showing the gate geometry and device principle is sketched in Fig. 1(a). We perform $S_{21}$ transmission measurements using a vector network analyzer and extract $f_{0}$ and quality factors. The resonator used here has a bare resonance frequency $f_{0}=4.86 \mathrm{GHz}$, a coupling quality factor $Q_{\mathrm{c}} \approx 1.4 \times 10^{5}$, and, importantly for this study, a high single photon internal quality factor $Q_{i} \approx 1.3 \times 10^{5}$. We probe the resonator at low powers corresponding to average photon numbers $\lesssim 100$ to avoid saturating the TLS.

\section{RESULTS AND DISCUSSION}

Figure 2 shows the variation in internal $Q_{i}$ versus applied gate voltage $V_{\mathrm{g}}$ as the resonance frequency is repeatedly detuned up to $\delta f \approx 64 \mathrm{MHz}$ (a single frequency sweep at a given gate voltage takes approximately 15 minutes, and each detuning step corresponds to approximately one resonance linewidth). We observe dips in these $Q(\delta f)$ traces; when sweeping both frequency and the gate voltage, these dips move in frequency and trace out hyperbolas on the $\delta f-V_{\mathrm{g}}$ plane, some reaching their minimum within the measurement window. Each of these hyperbolas originate from a strongly coupled coherent TLS with frequency

$$
f_{\mathrm{TLS}}\left(V_{\mathrm{g}}\right)=\sqrt{\Delta_{0}^{2}+\gamma^{2}\left(V_{\mathrm{g}}-V_{\mathrm{g} 0}\right)^{2}} / h,
$$

where $\gamma \sim \mathbf{p}_{0} / d_{\text {gate }}$ is the coupling strength of the TLS to the electric field induced by the applied gate voltage (see Appendix $\mathrm{B}$ for details), and $\mathbf{p}_{0}=q \mathbf{d}_{0}$ is the TLS dipole moment (with charge $q$ and displacement $d_{0}$ ) projected along the direction of the local electric field induced by the gate.

We now focus on the two segments (marked with red dashed lines in Fig. 2) with minima around $V_{\mathrm{g} 0}=-23 \mathrm{~V}$ and $-18 \mathrm{~V}$ and the same $\gamma$. Close inspection of Fig. 2 reveals that for each vertical trace the TLS is exclusively measured in one of two states only, a strong indication that these two hyperbolas originate from the same TLS.

The instability of TLS parameters is attributed to the switching of low energy TLFs [27] in the proximity of the TLS. One such TLF is dominant in this specific case and the switching rate is on the order of the sweep time, i.e., 15 minutes. Noteworthy, close to the hyperbola minimum the energies of the two states are degenerate and switching cannot be observed. This, together with the same $\gamma$ and minimum frequency of the two hyperbolas, indicates that the coupled 


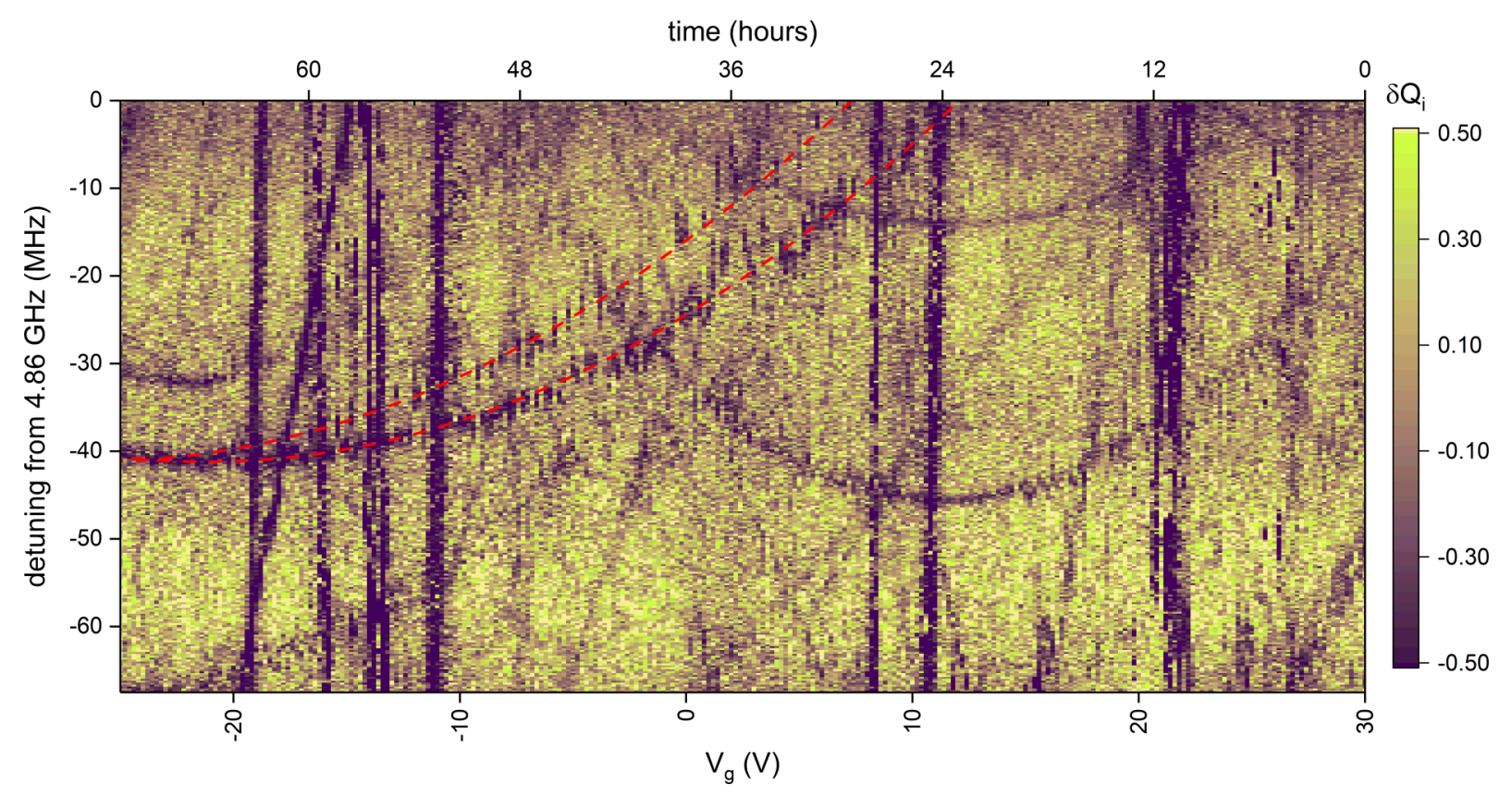

FIG. 2. Spectral and electric field mapping of TLSs. The measured variation of the quality factor $\delta Q_{i}=\left(Q_{i}-\left\langle Q_{i}\right\rangle\right) /\left\langle Q_{i}\right\rangle$ of a $4.86 \mathrm{GHz}$ resonator as a function of resonator frequency and applied gate voltage $\left(V_{\mathrm{g}}\right)$. Numerous individual TLS trace out the hyperbolas associated with additional loss (lower Q). Data is taken at $10 \mathrm{mK}$ and average photon number $\sim 10$.

TLF modifies the local electric field experienced by the TLS. The amount by which the fluctuating field perturbs the TLS energy depends on the TLS dipole moment $p_{0}$ and the TLFgenerated electric field at the TLS position.

To estimate $p_{0}$ we first extract the coupling strength $\gamma$ by fitting Eq. (1) to the hyperbola in Fig. 2 and find $\gamma=$ $19 \mathrm{MHz} / \mathrm{V}$. Next, for a known sample (and enclosure) geometry we can evaluate the typical electric field $E$ experienced by the majority of TLS if they were randomly distributed at the metal/air (MA) or substrate/air (SA) interfaces. Based on electrostatic simulations and the measured distribution of $\gamma$ 's from a large number of TLS hyperbolas we find that the most likely location for TLS with $\gamma$ on the order of $10-20 \mathrm{MHz}$ is on the MA interface and the most feasible dipole moment is $p_{0} \approx 1.9 \mathrm{e} \AA$ (see Appendix C), a quite plausible number for an atomically-scaled fluctuator. In what follows, we will assume that the incoherent TLFs are also hosted by structurally similar defects and therefore possess a characteristic dipole moment comparable with that of the TLS. Then the distance $r_{0}$ between the hopping TLS (cf. red dashed hyperbolas in Fig. 2) and the disturbing TLF can be estimated from the gate voltage offset $\delta V_{g 0}=5 \mathrm{~V}$ between the two hyperbolas. We find $r_{0} \approx$ $80 \mathrm{~nm}$ (Appendix C), which is consistent with the expected interaction volume $R^{3}$ of a TLS, previously found through analysis of ensemble effects to be $R \approx 100 \mathrm{~nm}$ at $10 \mathrm{mK}$ [16,27,36] (and scaling with temperature as $R \sim T^{-(1+\mu)}$, $\mu \approx 0.2$ being a parameter characterizing the $\alpha U^{\mu}$ energy dependence of the TLS density of states at low energies). The field modulation due to this particular TLF is likely on the upper scale of expected ensemble parameters, as no other switching TLS among about 50 studied so far shows similar or larger displacement, and we cannot exclude that for the majority of TLS the switching is masked by their linewidth. Accounting for possible screening effects would also yield a smaller $r_{0}$, although to accurately estimate screening at the atomic scale is not a straightforward task.

Counting the number of traces $n_{1}$ and $n_{2}$ in Fig. 2 that correspond to the TLS in each of two states we get the probability for the TLF to be found in its excited state, and from this probability we can estimate the asymmetry energy $\Delta_{0}$ for the TLF, $\Delta_{\mathrm{TLF}}: \ln \left(n_{1} / n_{2}\right)=-\Delta_{\mathrm{TLF}} / k_{\mathrm{B}} T$. Assuming the TLF temperature is that of the mixing chamber we find that $\Delta_{\mathrm{TLF}}=11 \mathrm{mK}(0.23 \mathrm{GHz})$, which is much smaller than $\Delta_{\mathrm{TLS}} / h=4.82 \mathrm{GHz}(230 \mathrm{mK})$.

If TLF switching disturbs the TLS energy by an amount similar to or less than the TLS linewidth, the above simple approach of counting traces in the two states could not be used. In this situation we instead trace the instantaneous resonator frequency using the Pound locking (PL) technique [37]. In principle, with the PL technique it is possible to trace the temporal resonance frequency variations with submillisecond resolution. However, here the observed TLF dynamics occurs at longer timescales which limits our sampling rate to $10 \mathrm{~Hz}$.

Combining the electrostatic gating and the frequency tuning we bring a strongly coupled TLS into resonance. Figure 3(a) presents the time-averaged resonator response when the gate-dependent TLS frequency crosses the resonance frequency. This particular TLS, when tuned through resonance, shifts the resonator frequency by $\sim \pm 40 \mathrm{kHz}$ (comparable to the resonator linewidth of $70 \mathrm{kHz}$ ). We measure the resonator frequency as a function of time at two selected values of the gate voltage $V_{\mathrm{g}}$ depicted in Fig. 3(a) with black and red dashed lines (here shown for the temperature $T=30 \mathrm{mK}$ ). The corresponding time series are shown in Fig. 3(b). Figure 3(c) shows the histograms of the same data. Off resonant with the TLS (black data) the histogram is well described by a single narrow Gaussian peak. Its width corresponds to the 'background' frequency noise of the resonator 
(a)

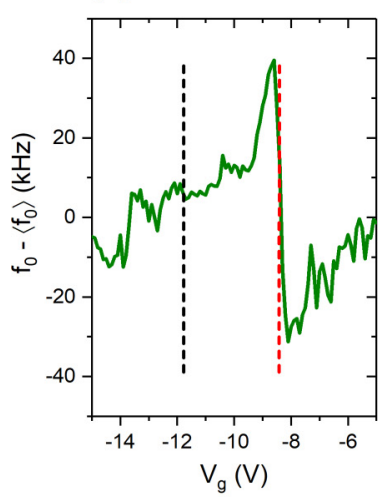

(b)

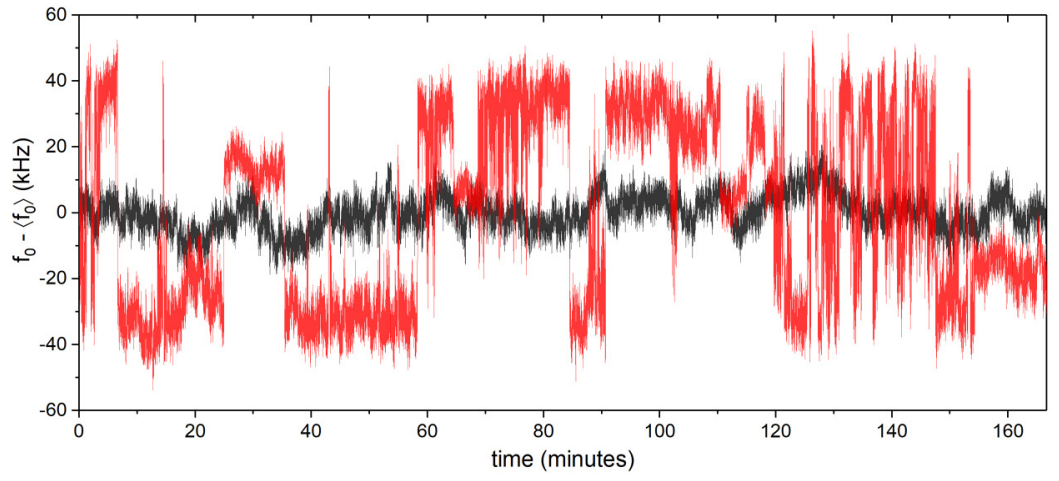

(c)

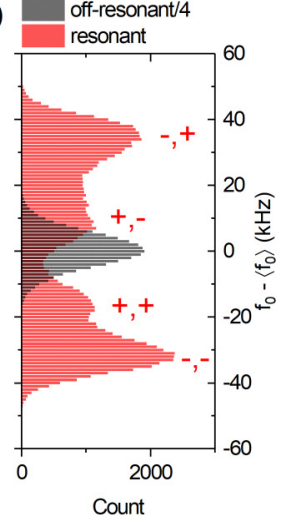

(d)

(e)

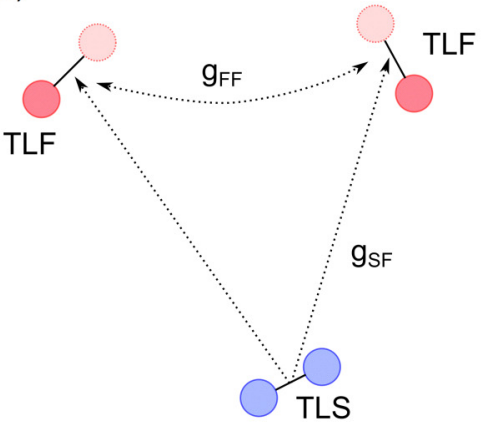

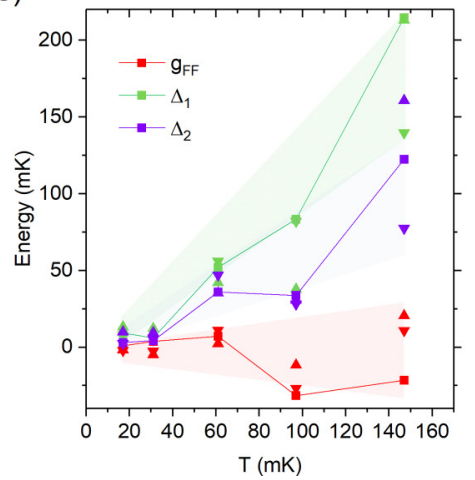

FIG. 3. (a) The time-averaged resonator frequency around its resonance with a strongly coupled TLS tuned by the gate. (b) Temporal fluctuations of the resonance frequency when resonator and TLS are tuned in (red) and off (black) resonance. Corresponding gate voltages are indicated by the dashed lines in (a). The measurements performed at $T=30 \mathrm{mK}$. (c) Histograms of the two time traces in (b). (d) Sketch of the corresponding microscopic TLF-TLS configuration discussed in the text. $g_{\mathrm{FF}}$ and $g_{\mathrm{SF}}$ show the couplings between the different entities. (e) The fitted TLF energy scales for a range of temperatures.

and a typical $1 / f$ noise power spectrum of magnitude similar to what was previously reported [16,36]; the background $1 / f$ noise stems from the energy drift of a large number of weakly coupled TLS. We then perform the same measurement on resonance with the strongly coupled TLS (red data). Its strong coupling allows us to single out the dynamics of one particular TLS from the rest of the TLS ensemble. Remarkably, we find four distinct peaks in the histogram of the $f_{0}(t)$ data.

The most straightforward interpretation of the four-peak histogram structure is that this particular TLS is strongly coupled to two low energy TLFs. The gate and frequency tunability in our experiment allows us to single out a dominant TLS coupled to multiple TLFs. This is direct evidence of multiple fluctuators coupled to the same TLS, whereas single ones have previously been identified [6]. Remarkably, the four-peak structure persists on histograms up to $T=100 \mathrm{mK}$ (see Appendix for more data), albeit less evident at high temperatures. At even higher temperatures ( $150 \mathrm{mK}$ and above) we observe irreversible thermal reconfiguration of the TLS [21] whereupon further measurements of the same TLS were not possible.

To always have just a few strongly coupled and thermally active TLFs would indeed be the expectation based on previous estimates $[16,27]$. In our particular case, based on the magnitude $A_{0}=3 \times 10^{-14}$ of the $1 / f$ fractional frequency noise $S_{y}(f)=A_{0} / f$ (extracted from the power dependence of the noise when off-resonant with a strongly coupled TLS) and the intrinsic loss tangent, we can make an order of magnitude estimate for the average expected number $N_{F}$ of thermally activated fluctuators coupled to each coherent TLS [16,27]. We find $N_{F}=c T^{1+\mu} \approx 5$ at $T=10 \mathrm{mK}$, where $c=1300 \mathrm{~K}^{-1-\mu}$ (see Appendix A). All four peaks in Fig. 3(c) are of similar amplitude, meaning that the TLS-TLF-TLF constellation spends roughly the same time in any of the four specific configurations. This indicates that all switching energies are on the same scale as the temperature, $30 \mathrm{mK}$. To further quantify this qualitative statement we note that in thermal equilibrium the occupation numbers $P_{ \pm, \pm}$for these four states are proportional to $e^{-U_{ \pm, \pm} / k_{\mathrm{B}} T}$, where the notation \pm indicates the two possible states of each TLF and $U_{ \pm, \pm}$are the energies of the corresponding conformation. The occupation numbers are given by the normalized peak areas in the measured histograms [Fig. 3(c)], $P_{ \pm, \pm}=A_{ \pm, \pm} / \Sigma A$ where $\Sigma A=A_{-,-}+$ $A_{+,-}+A_{-,+}+A_{+,+}$. The peak areas are obtained by fitting the histograms to four overlapping Gaussians (cf. Fig. 6 for the fit accuracy).

The microscopic picture of the interacting TLFs and TLS is sketched in Fig. 3(d). Assuming the TLF-TLS coupling $g_{\mathrm{SF}}$ is smaller than the TLF-TLF coupling $g_{\mathrm{FF}}$ (i.e., the backaction of the TLS on the TLF is negligible-an assumption which will be eventually justified by the numbers extracted from the fit), we can compose a Hamiltonian for the two-TLF 
subsystem as

$$
H=U_{0}-\frac{\Delta_{1}}{2} \sigma_{1}-\frac{\Delta_{2}}{2} \sigma_{2}-\frac{g_{\mathrm{FF}}}{2} \sigma_{1} \sigma_{2},
$$

where $\sigma_{1,2}$ indicate the states $( \pm)$ of the two TLFs, respectively. The ground $U_{-,-}$and highest energy $U_{+,+}$states can then straightforwardly be assigned to the most and least populated peaks, respectively. The assignment of $U_{+,-}$and $U_{-,+}$is merely a matter of notation. We thus arrive at a system of four equations $H( \pm, \pm)=-k_{\mathrm{B}} T \ln \left(P_{ \pm, \pm}\right)$which we solve for $\Delta_{1}$, $\Delta_{2}$, and $g_{\mathrm{FF}}$. From the data at $30 \mathrm{mK}$ in Figs. 3(b) and 3(c) we get $\Delta_{1}=12.0, \Delta_{2}=8.6$, and $g_{\mathrm{FF}}=3.9 \mathrm{mK}$.

The final ingredient in the microscopic picture in Fig. 3(d), the TLS-TLF coupling $g_{\mathrm{SF}}$, can be extracted from the observed shift in TLS frequency due to TLFs switchings, i.e., from the histogram peak-to-peak separation in Fig. 3(c). The $\sim 80 \mathrm{kHz}$ separation between the $(-,-)$ and $(+,-)$ peaks translates to an equivalent electric field increment of $\delta V_{\mathrm{g}}=$ $0.5 \mathrm{~V}$ [with the slope $\delta f\left(V_{\mathrm{g}}\right)$ in Fig. 3(a) as conversion factor], equivalent to $\delta E_{0} \sim 200 \mathrm{~V} / \mathrm{m}$. This corresponds to an interaction energy $E_{0} p_{0} \sim 0.5 \mathrm{mK}$, an order of magnitude below the extracted numbers for $g_{\mathrm{FF}}$. A posteriori, this justifies the previously made assumption that the TLS state exerts a negligible backaction on the TLFs. One possible interpretation for such a small number for the TLF-TLS coupling is that the TLS has a dipole moment ten times smaller than the TLF, though a more natural assumption would be that TLF-TLF distance is two times smaller than that of the two TLF-TLS distances (the dipole-dipole interaction scales as $\sim 1 / r^{3}$ ).

The same analysis was applied to the TLS switching data collected at a set of temperatures up to $150 \mathrm{mK}$ (cf. Appendix D: Fig. 6), the cumulative plot for asymmetry and interaction energies is shown in Fig. 3(e). It is quite clear that the higher the temperature the higher the characteristic energies of the TLFs disturbing the TLS. To understand this correlation we note that in a (idealized) glassy media one finds a hierarchy of fluctuators with arbitrary energy scales. At any specific temperature $T$ there exist fluctuators with switching energies $\Delta$ much lower, similar and higher than $T$. Fluctuators with $\Delta \ll k_{\mathrm{B}} T$ are switching too fast to be resolved as separate peaks in the histograms: TLFs with switching rates exceeding the measurement sample rate will contribute only to the generic $1 / f$ noise background and to the linewidth of the measured histograms. Only those TLFs with a switching rate commensurate with the bandwidth of the experiment can be observed as distinct peaks in the histograms. Varying the temperature we thus acquire information about the TLF spectral density. A set of histograms (Fig. 6) reveals that at any temperature this specific TLS is perturbed by just a few TLFs (one being dominant on some occasions), this means that the TLF spectral density is essentially uniform in the $10-100 \mathrm{mK}$ range.

We now turn to the nontrivial question of whether the measured data captures an ergodic representation of the full statistics of the system. As discussed above, at any temperature there exist high energy TLFs which fluctuate only on very long timescales, i.e., they may or may not flip during the given acquisition time. Such rare events can make histograms extracted from repetitive time traces poorly reproducible. To check for the reproducibility we perform the same histogram decomposition on the two halves of the acquired time traces; the $\Delta$ 's and $g_{F F}$ 's extracted from the subsets are depicted in Fig. 3(e) as triangles. We see that though the general energytemperature correlation holds, one cannot ascertain a specific functional dependence. Much more comprehensive statistics is needed to address the ergodicity issue and reveal the functional form of the temperature dependence of the TLF selfand interaction energies.

\section{CONCLUSIONS}

In summary, we have shown that using frequency tunable superconducting planar resonators in combination with electrostatic gating the spectral diffusion of individual two-level system defects can be mapped and their parameters obtained. More importantly, by utilizing the coupled TLS-resonator system as a sensor for the very low energy properties of the TLF bath we demonstrate the methodology for building a macroscopic picture of low energy glass physics based on individual observations. Such a route provides an alternative way to shed further light on the microscopic origin of low energy TLFs. These low energy defects are otherwise very challenging to study, yet they play an essential role in the generation of noise and decoherence in quantum devices. From a theoretical perspective, a number of long-standing conundrums remain unanswered for amorphous glassy media. In particular, the "universality problem" [38-40] and the failure of the STM for some materials below $\sim 100 \mathrm{mK}[34,40$ 42]. This has stimulated attempts to address these problems by including TLF interactions in the form of inversion asymmetry [33], dipole [27,32] or quadrupole [43,44] interactions, or TLS clustering $[42,45]$. The methods presented here are likely to aid in identifying appropriate models [34] for, and the origin of, these elusive microscopic defects. Through detailed temperature dependence of the TLF switching behavior and the statistics gathered on a large number of TLS/TLF constellations, the TLF energy density spectrum and the dominant switching mechanism $[46,47]$ can be deduced. Such information will provide very restrictive selection criteria on a plethora of phenomenological TLF models discussed in literature [5]. Through clear experimental evidence for two strongly coupled fluctuators to the same TLS we are able to extract TLF-TLF interaction energy which remains consistently small $(\leqslant 10 \mathrm{mK})$ for a range of temperatures. Interestingly, the ascertained energy scales and the uniformity of the TLF spectrum are commensurate with the conjectured model for the ubiquitous $1 / f$ flux noise in quantum devices $[2,48,49]$.

\section{ACKNOWLEDGMENTS}

We thank T. Lindström and D. Golubev for useful discussions and V. Ranjan for careful reading of the paper. Samples were fabricated in the nanofabrication facilities of the department of microtechnology and nanoscience at Chalmers university. We acknowledge the support of the UK government department for Business, Energy and Industrial Strategy through the UK national quantum technologies programme. S.K. and A.D. acknowledge support from the Swedish Research Council (VR) (Grant Agreement Nos. 2016-04828 and 2019-05480), EU H2020 European 
Microkelvin Platform (Grant Agreement No. 824109), the EU Horizon 2020 research and innovation programme (Grant Agreement No. 766714/HiTIMe), and Chalmers Area of Advance NANO/2018.

\section{APPENDIX A: ESTIMATING $N_{F}$ FROM THE $1 / f$ FREQUENCY NOISE AMPLITUDE}

From the generalized tunneling model [27] (a model descendant from the STM which takes into account TLS interactions), we can estimate the average number of thermally active TLFs coupled to a specific TLS, $N_{F}$, from the measured loss tangent $\chi \approx \tan \delta_{i}$ and magnitude of the $1 / f$ frequency noise $A_{0}$ :

$$
A_{0}=\pi \chi^{2} F^{2} P_{\gamma} U_{0} \frac{N_{F}}{\Gamma_{2} V_{h}} .
$$

Here $F$ is a geometric filling factor, $P_{\gamma}$ is a constant of order unity that depends logarithmically on TLF switching rates, $U_{0}=p_{0}^{2} / \varepsilon_{0} \varepsilon_{r}$, and $h \Gamma_{2}=\ln \left(\Gamma_{\max } / \Gamma_{\min }\right) \chi\left(k_{\mathrm{B}} T\right)^{1+\mu} /\left(h f_{0}\right)^{\mu}$ is the average TLF linewidth. The average number of active fluctuators coupled to each TLS is then

$$
N_{F}=\frac{A_{0} h \Gamma_{2} V_{h} \varepsilon_{0} \varepsilon_{r}}{\pi\left(F \tan \delta_{i}\right)^{2} P_{\gamma} p_{0}^{2}} .
$$

We use typical parameters [16] $P_{\gamma}=0.5, \varepsilon_{r}=10$, the logarithm of the ratio of the minimum and maximum of the switching rate distribution $\ln \left(\Gamma_{\max } / \Gamma_{\min }\right)=20$, and $\mu=$ 0.2 . The device specific parameters are [16,21] $F \tan \delta_{i}=$ $0.7 \times 10^{-5}, V_{h} \approx 2 \times 10^{-16} \mathrm{~m}^{3}, f_{0}=4.86 \mathrm{GHz}, F=0.015$ which results in $N_{F}=c T^{1+\mu} \approx 5$ at $T=10 \mathrm{mK}$, where $c=$ $1300 \mathrm{~K}^{-1-\mu}$.

\section{APPENDIX B: GATE-INDUCED ELECTRIC FIELD STRENGTH}

Figure 4(a) shows the detailed geometry of the sample and its enclosure with the embedded gate electrode used to tune the TLS energy via the electric field induced on the TLS by the gate potential. All metal structures on the sample (superconducting) are connected to the ground potential with a negligible resistance. The resulting static electric field strength at the device surface is shown in Fig. 4(b), evaluated using COMSOL. The figure shows the electric field strength induced by the top gate electrode at the metal-air (MA), metal-substrate (MS), and substrate-air (SA) interfaces for a representative cross section of the device. Figure 4(c) shows the probability distributions $P(E)$ of finding a TLS randomly placed at the MA- (red histogram), the MS- (blue histogram), or the SA- (orange histogram) interfaces at a point where the gate-induced electric field has a specific value $E$. The histograms were obtained from a 3D electrostatics model solved for one elementary cell of the quasifractal device structure (see Ref. [25] for design details), with a surface mesh element size of $1 \mathrm{~nm}^{2}$. The gate electrode is much larger than the resonator structure, hence the field strength is assumed to be equal for all elementary cells across the device. (a)

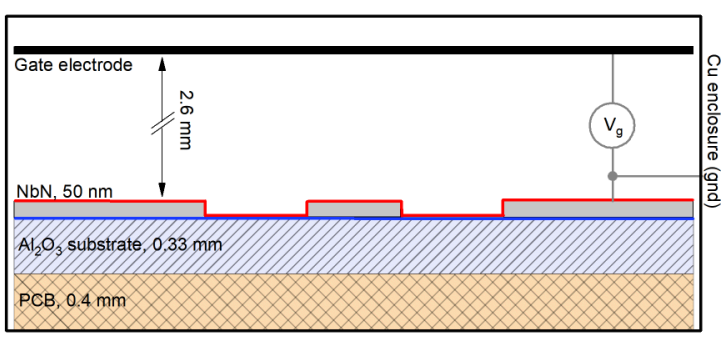

(b)

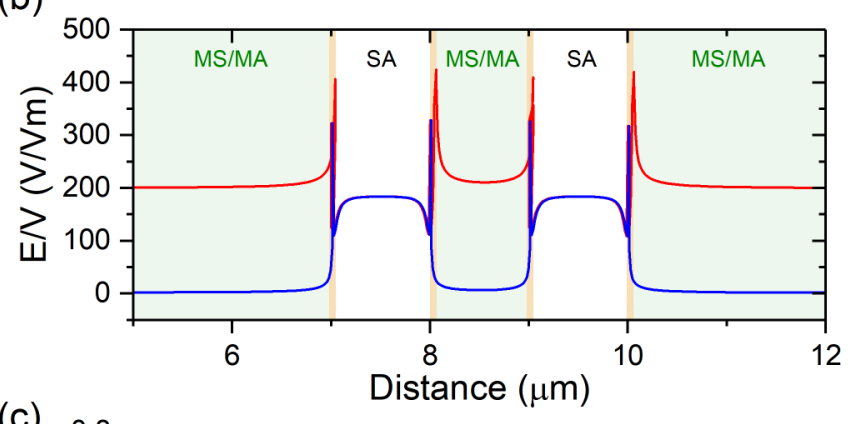

(c)

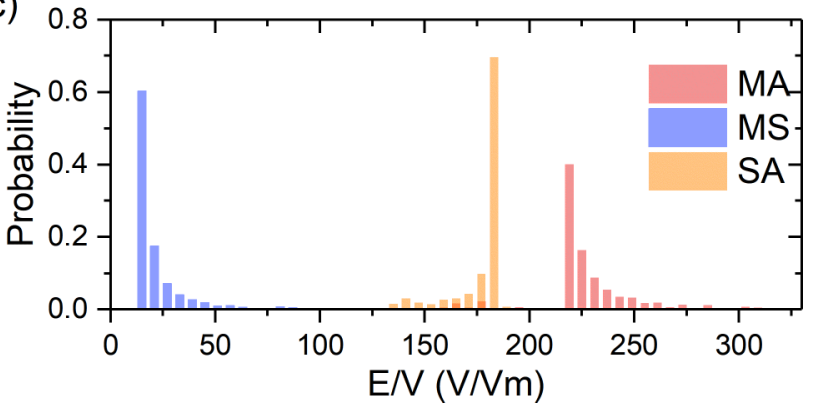

FIG. 4. (a) Schematic (not to scale) of the sample and its enclosure outlining the location of the gate electrode and ground potentials with respect to the sample. (b) Simulated electric field strength (for $1 \mathrm{~V}$ applied to the gate) along the two surfaces indicated by the red and blue contours in (a). (c) Normalized histogram showing the probability of finding a TLS, randomly placed at the MS or MA interfaces, at a point with a certain electric field strength.

\section{APPENDIX C: TLS PARAMETER DISTRIBUTIONS}

From the calculated $P(E)$, which gives the likelihood of finding a particular electric field strength anywhere on the device surface, we evaluate the dipole moment of the observed TLS with $\gamma=19 \mathrm{MHz} / \mathrm{V}$ in Fig. 2 using $p_{0}=$ $(\gamma h / 2)(\partial V / \partial E)$. This gives the probability distribution for the dipole moment of this particular TLS shown in Fig. 5(a), which is peaked around $1.9 \mathrm{e} \AA$. Here we have neglected the contribution from the MS interface, as this would yield dipole moments that are incommensurate with the rest of our observations. The MA and SA interfaces are almost indistinguishable in terms of $E$-field strengths and are here considered jointly.

Next, the distance between the TLS and the TLF which it is coupled to can be estimated from the offset of the two highlighted hyperbolas in Fig. 2 using

$$
\frac{4 \pi}{e} \varepsilon_{r} \varepsilon_{0} \delta E=\frac{1}{\left(r_{0}-\delta r \cos \theta\right)^{2}}-\frac{1}{\left(r_{0}+\delta r \cos \theta\right)^{2}},
$$



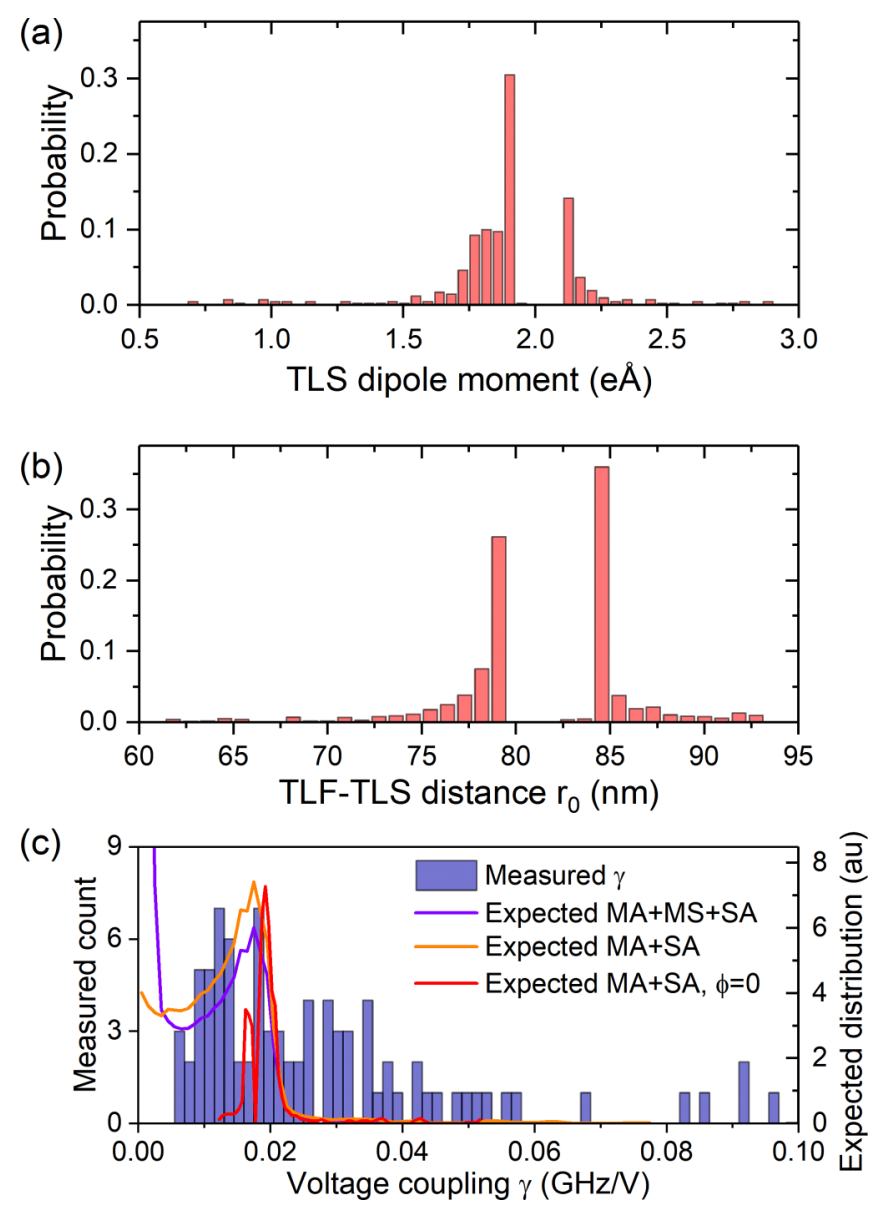

FIG. 5. (a) Assuming the TLS is located at either the MA or SA interface we can from the fitted hyperbola and the $E$-field distribution obtain a probability distribution for the dipole moment of the TLS. (b) The probability distribution of the TLF-TLS separation $r_{0}$ assuming the rotation angle $\phi=0$. A larger $\phi$ would shift the distribution towards smaller $r_{0}$. (c) Measured distribution of TLS voltage coupling coefficients $\gamma$ obtained by fitting a large number of hyperbolas observed across a number of cooldowns. Only hyperbolas that are well defined (e.g., by the TLS minimum energy within or near the measured frequency window) are included in the statistics. The solid lines show the expected distribution obtained from the surface $E$-field distribution and assuming a uniform angular distribution of TLS orientations.

where $\delta r=p_{0}^{\mathrm{TLF}} / 2 e, \theta$ accounts for the projection of the TLF displacement towards the direction of the TLS, and $\delta E$ is the local variation in electric field deduced from the offset of the two parabolas in Fig. 2. The TLF is assumed to have the same dipole moment as the TLS. Solving for $r_{0}$ with $\phi=0$ we can evaluate the probability distribution in $r_{0}$, which is peaked around $r_{0} \approx 78 \mathrm{~nm}$. This distribution is shown in Fig. 5(b) and takes into account the MA and SA interfaces only.

Finally, we show that the measured $\gamma=19 \mathrm{MHz} / \mathrm{V}$ is commensurate with a TLS on the MA surface of our device, located away from any metal edges. Close to the edges the electric field is enhanced and a TLS placed there should have a much stronger gate response. We associate those near-edge TLS with the almost vertical lines that can be seen in Fig. 2.

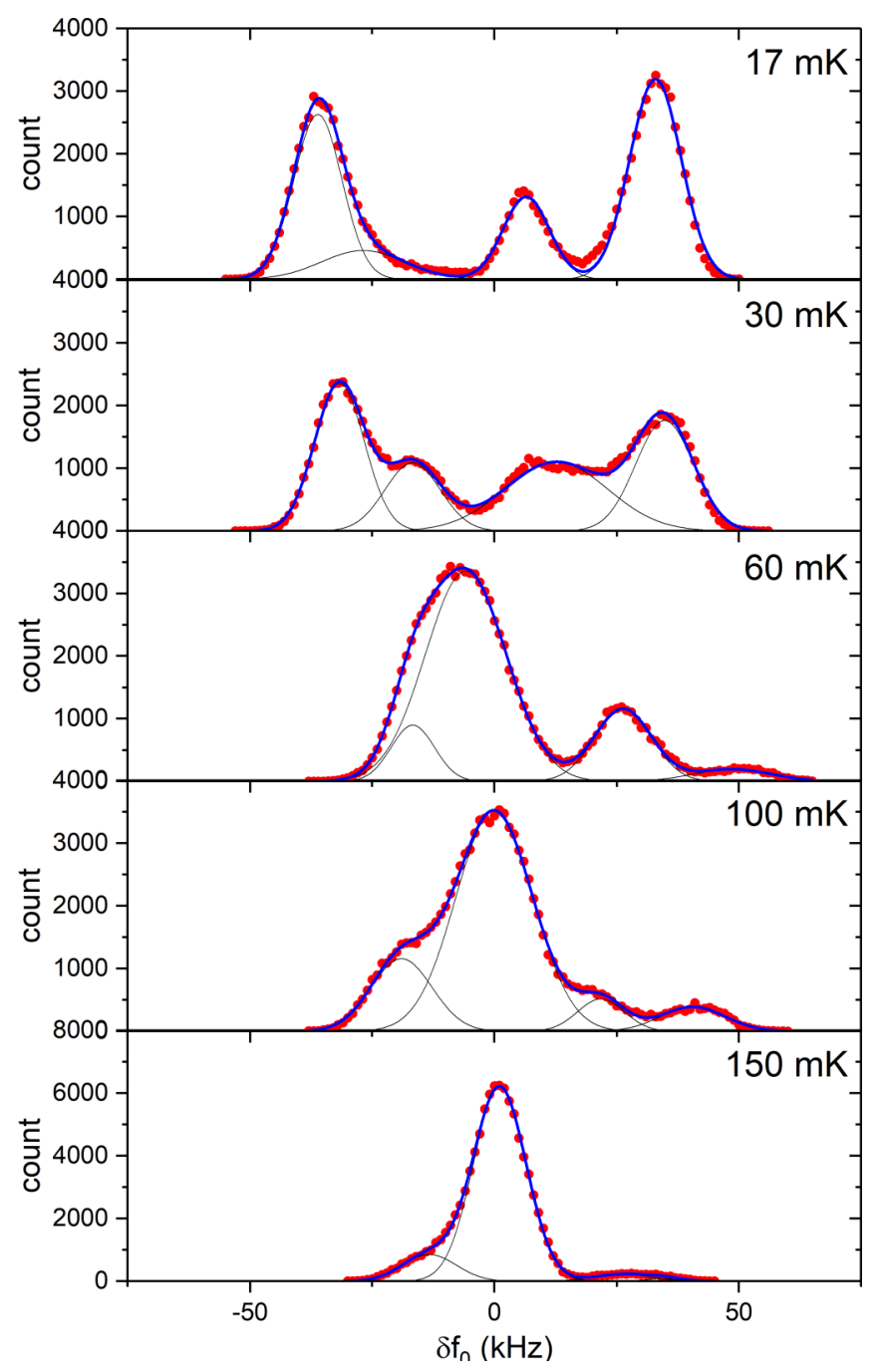

FIG. 6. Distribution of resonator frequency for the coupled resonator-TLS-TLF system together with Gaussian peak fits at different temperatures.

These segments can be fitted with $\gamma$ 's that are 20-50 times larger, in agreement with the enhanced electric fields near the metal edges. Excluding these from further analysis, we focus on the TLS that have clear, observable hyperbolas.

Figure 5(c) shows the histogram of extracted coupling coefficients from a large number of individual such TLS hyperbolas seen in a number of measurements (several devices/cooldowns). Naively one would expect that this distribution reflects the $E$-field distribution of the device [Fig. 4(c)], albeit averaged across all TLS angles $\theta$, stretching the distribution towards lower $\gamma$. For a uniform distribution of TLS angles $\theta$ we would expect the distribution in $\gamma$ to continue down to zero. This expected distribution using the assumption of $p_{0}=1.9 \mathrm{e} \AA$ for two sets of surfaces considered $(\mathrm{MA}+\mathrm{SA}$ and $\mathrm{MA}+\mathrm{SA}+\mathrm{MS})$ is shown as the solid lines in Fig. 5(c). Interestingly, the measured distribution does not continue smoothly down to $\gamma=0$, but instead it is abruptly terminated with no $\gamma \lesssim 8 \mathrm{MHz} / \mathrm{V}$. This implies that none of the TLS observed are located at the MS interface where the gate is well screened. 
However, $\theta=\pi$ implies that the TLS dipole moment is orthogonal to both the DC gate and the RF electric field. Thus the coupling strength to the resonator vanishes simultaneously, explaining why we do not observe any TLS with $\gamma=0$ at the MA interface. At the SA interface this assumption does not hold: RF fields are predominantly in the surface plane but DC fields have a non-negligible perpendicular component. Likewise, well inside the MS interface we have very small DC fields that are orthogonal to the metal surface where the RF field is still strong. Hence we conclude that the majority of the TLS we observe are on (or very near) the metal surface. In addition it would be natural to have a distribution of dipole moments, further smearing the expected $\gamma$ distribution. The theory expectation in Fig. 5(c) is calculated assuming a maximum dipole moment projection of $1.9 \mathrm{e} \AA$ and mainly highlights the lack of TLS observed with low $\gamma$. The data also shows more TLS with larger $\gamma$ than what is to be expected from this estimate. This is likely the result of TLS defects having a distribution of dipole moments and different locations within the interfaces that results in varying degrees of screening of the local electric field.

\section{APPENDIX D: SWITCHING HISTOGRAMS AT DIFFERENT TEMPERATURES}

In Fig. 6 we show the switching statistics obtained for the coupled TLF-TLF-TLS-resonator system at different temperatures together with the Gaussian fits used to extract the peak areas. Each dataset spans 2.8 hours of observations gaplessly sampled at a rate of $10 \mathrm{~Hz}$.
[1] F. Arute, K. Arya, R. Babbush, D. Bacon, J. C. Bardin, R. Barends, R. Biswas, S. Boixo, F. G. S. L. Brandao, D. A. Buell et al., Quantum supremacy using a programmable superconducting processor, Nature (London) 574, 505510 (2019).

[2] E. Paladino, Y. M. Galperin, G. Falci and B. L. Altshhuler, 1/f noise: Implications for solid-state quantum information, Rev. Mod. Phys. 86, 361 (2014).

[3] P. V. Klimov, J. Kelly, Z. Chen, M. Neeley, A. Megrant, B. Burkett, R. Barends, K. Arya, B. Chiaro, Y. Chen et al., Fluctuations of Energy-Relaxation Times in Superconducting Qubits, Phys. Rev. Lett. 121, 090502 (2018).

[4] J. Burnett, A. Bengtsson, M. Scigliuzzo, D. Niepce, M. Kudra, P. Delsing, and J. Bylander, Decoherence benchmarking of superconducting qubits, npj Quantum Inf. 5, 54 (2019).

[5] C. Müller, J. H. Cole, and J. Lisenfeld, Towards understanding two-level-systems in amorphous solids - insights from quantum devices, Rep. Prog. Phys. 82, 124501 (2019).

[6] S. M. Meißner, A. Seiler, J. Lisenfeld, A. V. Ustinov, and G. Weiss, Probing individual tunneling fluctuators with coherently controlled tunneling systems, Phys. Rev. B 97, 180505(R) (2018).

[7] G. J. Grabovskij, T. Peichl, J. Lisenfeld, G. Weiss, and A. V. Ustinov, Strain tuning of individual atomic tunneling systems detected by a superconducting qubit, Science 338, 232 (2012).

[8] J. M. Martinis, K. B. Cooper, R. McDermott, M. Steffen, M. Ansmann, K. D. Osborn, K. Cicak, S. Oh, D. P. Pappas, R. W. Simmonds, and C. C. Yu, Decoherence in Josephson Qubits from Dielectric Loss, Phys. Rev. Lett. 95, 210503 (2005).

[9] J. Lisenfeld, G. J. Grabovskij, C. Muüller, J. H. Cole, G. Weiss, and A. V. Ustinov, Observation of directly interacting coherent two-level systems in an amorphous material, Nat. Commun. 6, 6182 (2015).

[10] J. Lisenfeld, A. Bilmes, A. Megrant, R. Barends, J. Kelly, P. Klimov, G. Weiss, J. M. Martinis, and A. V. Ustinov, Electric field spectroscopy of material defects in transmon qubits, npj Quantum Information 5, 105 (2019).

[11] M. J. A. Stoutimore, M. S. Khalil, C. J. Lobb, and K. D. Osborn, A Josephson junction defect spectrometer for measuring twolevel systems, Appl. Phys. Lett. 101, 062602 (2012).

[12] A. Bilmes, A. Megrant, P. Klimov, G. Weiss, J. M. Martinis, A. V. Ustinov, and J. Lisenfeld, Resolving the positions of defects in superconducting quantum bits, Sci. Rep. 10, 3090 (2020).

[13] A. Bilmes, S. Volosheniuk, J. D. Brehm, A. V. Ustinov and J. Lisenfeld, Quantum sensors for microscopic tunneling systems, npj Quantum Inf. 7, 27 (2021).

[14] V. Ranjan, S. Probst, B. Albanese, T. Schenkel, D. Vion, D. Esteve, J. Morton, and P. Bertet, Electron spin resonance spectroscopy with femtoliter detection volume, Appl. Phys. Lett. 116, 184002 (2020).

[15] S. E. de Graaf, A. A. Adamyan, T. Lindström, D. Erts, S. E. Kubatkin, A. Ya. Tzalenchuk and A. V. Danilov, Direct Identification of Dilute Surface Spins on $\mathrm{Al}_{2} \mathrm{O}_{3}$ : Origin of Flux Noise in Quantum Circuits, Phys. Rev. Lett. 118, 057703 (2017).

[16] S. E. de Graaf, L. Faoro, J. Burnett, A. A. Adamyan, A. Ya. Tzalenchuk, S. E. Kubatkin, T. Lindström and A. V. Danilov, Suppression of low-frequency charge noise in superconducting resonators by surface spin desorption, Nat. Commun. 9, 1143 (2018).

[17] M. S. Moeed, C. T. Earnest, J. H. Béjanin, A. S. Sharafeldin, and M. Mariantoni, Improving the time stability of superconducting planar resonators, MRS Advances 4, 2201 (2019).

[18] S. Un, S. E. de Graaf, P. Bertet, S. E. Kubatkin, and A. V. Danilov (unpublished).

[19] J. Burnett, L. Faoro, I. Wisby, V. L. Gurtovoi, A. V. Chernykh, G. M. Mikhailov, V. A. Tulin, R. Shaikhaidarov, V. Antonov, P. J. Meeson, A. Ya. Tzalenchuk, and T. Lindström, Evidence for interacting two-level systems from the 1/f noise of a superconducting resonator, Nat. Commun. 5, 4119 (2014).

[20] S. E. de Graaf, A. Ya. Tzalenchuk, and T. Lindström, 1/f frequency noise of superconducting resonators in large magnetic fields, Appl. Phys. Lett. 113, 142601 (2018).

[21] S. E. de Graaf, L. Faoro, L. B. Ioffe, S. Mahashabde, J. J. Burnett, T. Lindström, S. E. Kubatkin, A. V. Danilov, and A. Ya. Tzalenchuk, Two-level systems in superconducting quantum devices due to trapped quasiparticles, Science Advances 6, eabc5055 (2020).

[22] C. R. H. McRae, H. Wang, J. Gao, M. Vissers, T. Brecht, A. Dunsworth, D. Pappas, and J. Mutus, Materials loss measurements using superconducting microwave resonators, Rev. Sci. Instrum. 91, 091101 (2020). 
[23] J. D. Brehm, A. Bilmes, G. Weiss, A. V. Ustinov, and J. Lisenfeld, Transmission-line resonators for the study of individual two-level tunneling systems, Appl. Phys. Lett. 111, 112601 (2017).

[24] B. Sarabi, A. N. Ramanayaka, A. L. Burin, F. C. Wellstood, and K. D. Osborn, Projected Dipole Moments of Individual Two-Level Defects Extracted Using Circuit Quantum Electrodynamics, Phys. Rev. Lett. 116, 167002 (2016).

[25] S. Mahashabde, E. Otto, D. Montemurro, S. E. de Graaf, S. E. Kubatkin, and A. V. Danilov, Fast Tunable High Q-Factor Superconducting Microwave Resonators, Phys. Rev. Applied 14, 044040 (2020).

[26] D. Niepce, J. J. Burnett, M. Kudra, J. H. Cole, and J. Bylander, Stability of superconducting resonators: motional narrowing and the role of Landau-Zener driving of two-level defects, arXiv:2008.07038.

[27] L. Faoro and L. B. Ioffe, Interacting tunneling model for twolevel systems in amorphous materials and its predictions for their dephasing and noise in superconducting microresonators, Phys. Rev. B 91, 014201 (2015).

[28] L. Faoro, J. Bergli, B. L. Altshuler, and Y. M. Galperin, Models of Environment and $T_{1}$ Relaxation in Josephson Charge Qubits, Phys. Rev. Lett. 95, 046805 (2005).

[29] L. Faoro and L. B. Ioffe, Quantum Two Level Systems and Kondo-Like Traps as Possible Sources of Decoherence in Superconducting Qubits, Phys. Rev. Lett. 96, 047001 (2006).

[30] J. Lisenfeld, A. Bilmes, S. Matityahu, S. Zanker, M. Marthaler, M. Schechter, G. Schön, A. Shnirman, G. Weiss, and A. V. Ustinov, Decoherence spectroscopy with individual two-level tunneling defects, Sci. Rep. 6, 23786 (2016).

[31] W. A. Phillips, Two-level states in glasses, Rep. Prog. Phys. 50, 1657 (1987).

[32] A. L. Burin, S. Matityahu, and M. Schechter, Low-temperature 1/f noise in microwave dielectric constant of amorphous dielectrics in Josephson qubits, Phys. Rev. B 92, 174201 (2015).

[33] M. Schechter, and P. C. E. Stamp, Inversion symmetric two-level systems and the low-temperature universality in disordered solids, Phys. Rev. B 88, 174202 (2013).

[34] C. C. Yu and H. M. Carruzzo, Two-level systems and the tunneling model: A critical view, arXiv:2101.02787.

[35] M. R. Vissers, J. Hubmayr, M. Sandberg, S. Chaudhuri, C. Bockstiegel, and J. Gao, Frequency-tunable superconducting resonators via nonlinear kinetic inductance, Appl. Phys. Lett. 107, 062601 (2015).

[36] J. Burnett, L. Faoro and T. Lindström, Analysis of high quality superconducting resonators: consequaneces for TLS properties in amorphous oxides, Supercond. Sci. Technol. 29, 044008 (2016).

[37] T. Lindström, J. Burnett, M. Oxborrow, and A. Ya. Tzalenchuk, Pound-locking for characterization of superconducting microresonators, Rev. Sci. Instrum. 82, 104706 (2011).

[38] R. O. Pohl, X. Liu, and E. Thompson, Low-temperature thermal conductivity and acoustic attenuation in amorphous solids, Rev. Mod. Phys. 74, 991 (2002).

[39] V. Lubchenko, Low-temperature anomalies in disordered solids: a cold case of contested relics? Adv. Phys.: X 3, 1510296 (2018).

[40] M. Schechter, P. Nalbach, and A. L. Burin, Nonuniversality and strongly interacting two-level systems in glasses at low temperatures, New J. Phys. 20, 063048 (2018).

[41] M. A. Ramos, Are universal "anomalous" properties of glasses at low temperatures truly universal? Low Temp. Phys. 46, 104 (2020).

[42] D. Ding, D. van Driel, L. M. C. Pereira, J. F. Bauters, M. J. R. Heck, G. Welker, M. J. A. de Dood, A. Vantomme, J. E. Bowers, W. Löffler, and D. Bouwmeester, Probing interacting twolevel systems with rare-earth ions, Phys. Rev. B 101, 014209 (2020).

[43] A. Würger, A. Fleischmann, and C. Enss, Dephasing of Atomic Tunneling by Nuclear Quadrupoles, Phys. Rev. Lett. 89, 237601 (2002).

[44] M. Bartkowiak, M. Bazrafshan, C. Fischer, A. Fleischmann, and C. Enss, Nuclear Quadrupole Moments as a Microscopic Probe to Study the Motion of Atomic Tunneling Systems in Amorphous Solids, Phys. Rev. Lett. 110, 205502 (2013).

[45] A. L. Burin and Y. Kagan, The low energy collective excitations in dielectric glasses. The attenuation of sound waves at ultra low temperatures, Zh. Eksp. Teor. Fiz. 107, 633 (1995) [JETP 80, 761 (1995)].

[46] A. J. Leggett, S. Chakravarty, A. T. Dorsey, M. P. A. Fisher, A. Garg and W. Zwerger, Dynamics of the dissipative two-state system, Rev. Mod. Phys. 59, 1 (1987).

[47] N. V. Prokof'ev and P. C. E. Stamp, Theory of the spin bath, Rep. Prog. Phys. 63, 669 (2000).

[48] T. Lanting, M. H. Amin, C. Baron, M. Babcock, J. Boschee, S. Boixo, V. N. Smelyanskiy, M. Foygel, and A. G. Petukhov, Probing environmental spin polarization with superconducting flux qubits, arXiv:2003.14244.

[49] A. De, 1/f flux noise in low- $T_{c}$ SQUIDs due to superparamagnetic phase transitions in defect clusters, Phys. Rev. B 99, 024305 (2019). 